九州大学学術情報リポジトリ

Kyushu University Institutional Repository

\title{
Improvement of Faugère et al.' s method to solve ECDLP
}

Yun-Ju, Huang

Graduate School of Mathematics, Kyushu University

Petit, Christophe

UCL Crypto Group

Shinohara, Naoyuki

NICT

Takagi, Tsuyoshi

Institute of Mathematics for Industry, Kyushu University

http://hdl. handle. net/2324/1434304

出版情報 : MI lecture note series. 53，pp.145-158，2013-12-26. 九州大学マス・フォア・インダスト リ研究所

バージョン：

権利関係 : 


\title{
Improvement of Faugère et al.'s method to solve ECDLP
}

\author{
$\circ$ Huang Yun-Ju* \\ Christophe Petit * \\ Naoyuki Shinohara ${ }^{\dagger}$ \\ Tsuyoshi Takagi $\ddagger$ \\ *Graduate School of Mathematics, Kyushu University \\ * UCL Crypto Group \\ $\dagger$ NICT \\ $\ddagger$ Institute of Mathematics for Industry, Kyushu University
}

August 29, 2013

\section{Abstract}

- Target : ECDLP problem.

- Motivation : A new technique for index calculus method algorithm to solve ECDLP proposed by Faugère et al. at Eurocrypt 2012.

- Contribution :

1. Give a new idea to improve the algorithm proposed by Faugère et al.

2. Implements different strategies solving ECDLP and compares them. 


\section{Outline}

\section{Target - ECDLP}

Background

Index Calculus Method with Gröbner Basis

Our Contribution

\section{Elliptic Curve Discrete Log Problem (ECDLP)}

Let $F_{2^{n}}$ is a binary field of prime degree $n$ over $F_{2}$.

Let $E_{\alpha, \beta}: y^{2}+x y=x^{3}+\alpha x^{2}+\beta$ over field $F_{2^{n}}$, where $\alpha, \beta \in F_{2^{n}}$.

Given $P \in E_{\alpha, \beta}, Q \in\langle P\rangle$,

\section{Target}

Find smallest non-negative integer $k$ such that $Q=[k] P$ 


\section{Known Algorithm}

- Exhaustive Search

Time Complexity : $\mathrm{O}\left(2^{n}\right)$

- Pollard-rho Mehod

Time Complexity : $\mathrm{O}\left(2^{\frac{n}{2}}\right)$

- Index Calculus Method

Time Complexity : claimed to be sub-exponential

$\mathrm{O}\left(2^{c n^{2 / 3} \log n}\right)$ by Petit et al. at Asiacrypto 2012.

\section{Generic Index Calculus Mehod}

Generic Index Calculus Method

Input :

$P, Q \in E_{\alpha, \beta}$

Output :

$k \in N$ such that $Q=[k] P$

phase 1:

Setup factor base $F_{V}=\left\{P_{i} \in E_{\alpha, \beta} \mid x\left(P_{i}\right) \in V\right\}$

phase 2: Relation Search

Find sufficient relations

sol $_{m}=\left\{\sum_{1 \leq j \leq m} P_{j}^{\prime}=[a] P+[b] Q\right\}$

for random $a, b \in N, P_{j}^{\prime} \in F_{V}$.

phase 3:

Transform the relation to matrix $M$.

phase 4:

Find reduced echelon form $M_{-}$of $M$.

phase 5:

Solve the relation $\left[a^{\prime}\right] P+\left[b^{\prime}\right] Q=O$ in $M_{\text {. }}$. $k=\frac{-a^{\prime}}{b^{\prime}}$.

$x\left(P_{i}\right)$ means $x$-coordinate of $P_{i}$. 


\section{Generic Index Calculus}

Points of $E_{\alpha, \beta}$



Generic Index Calculus Method

$$
\begin{aligned}
& \begin{array}{llllll}
P_{1} & P_{2} & \ldots & P_{\# F_{V}} & P & Q
\end{array} \\
& \left(\begin{array}{cccccc}
1 & 1 & & 0 & a_{1} & b_{1} \\
1 & 0 & & 0 & a_{2} & b_{2} \\
\vdots & & \ddots & \vdots & & \vdots \\
& & & & & \\
1 & 1 & \ldots & 1 & a_{t} & b_{t}
\end{array}\right) \\
& \Downarrow \text { reduced row echelon form } \\
& \left(\begin{array}{cccccc}
1 & 0 & & 0 & & \\
0 & 1 & & 0 & & \\
\vdots & & \ddots & \vdots & & \\
& & & & & \\
0 & 0 & \ldots & 1 & & \\
0 & 0 & & 0 & a^{\prime} & b^{\prime}
\end{array}\right)
\end{aligned}
$$




\section{Semaev's Polynomials[1]}

Property - Semaev's summation polynomial

For $R=[a] P+[b] Q$, Semaev's summation polynomials $s_{m+1}$ are multivariate polynomials where:

$\forall x_{1}, \ldots, x_{m} \in F_{2^{n}}$

$$
s_{m+1}\left(x_{1}, x_{2}, \ldots, x_{m}, x_{r}\right)=0
$$

if and only if $\exists P_{j}^{\prime}, 1 \leq j \leq m$ such that

$$
\sum_{1 \leq j \leq m} P_{j}^{\prime}+R=O
$$

where $x_{j}=x\left(P_{j}^{\prime}\right), x_{r}=x(R)$.

The problem to find $P_{j}^{\prime}$ s.t, $\sum P_{j}^{\prime}=R$ is now reduced to solve $s_{m+1}\left(x_{1}, x_{m}, x_{r}\right)=0 . x_{j}$ is variable and $x_{r}$ is known value.

\section{Version by Faugère et al. (FPPR)}

In Eurocrypt 2012, Faug'ere, Perret, Petit and Renault proposed a new version to solve the Semaev's summation polynomials by Gröbner basis for phase 2 (Relation Search). 


\section{Variable Rewritten}

We can regard $F_{2^{n}}$ as the vector space defined by the basis $\left\{v_{0}, v_{1}, \ldots, v_{n^{\prime}-1}\right\}$.

Variable Substitution

Let $x_{j}=x\left(P_{j}^{\prime}\right), P_{j}^{\prime} \in F_{v}$, if we rewrite $x_{j}=\sum_{0 \leq \ell \leq n^{\prime}-1} c_{j, l} v_{\ell}$, then

$s_{m+1}\left(x_{1}, \ldots, x_{m}, x_{r}\right)$

$=s_{m+1}\left(\sum_{0 \leq \ell \leq n^{\prime}-1} c_{1, \ell} v_{\ell}, \ldots, \sum_{0 \leq \ell \leq n^{\prime}-1} c_{m, \ell} v_{\ell}, \sum_{0 \leq 1 \leq n-1} r_{\ell} v_{\ell}\right)$

where $c_{j, \ell} \in F_{2}$ is unknown and $r_{\ell}$ is known.

\section{Multivariable Polynomial System}

Multivariable Polynomial System

$s_{m+1}\left(\sum_{0 \leq \ell \leq n^{\prime}-1} c_{1, \ell} v_{\ell}, \ldots, \sum_{0 \leq I \leq n^{\prime}-1} c_{m, \ell} v_{\ell}, \sum_{0 \leq \ell \leq n-1} r_{\ell} v_{\ell}\right)$

$=f_{0}\left(c_{j, \ell}\right) v_{1}+f_{1}\left(c_{j, \ell}\right) v_{2}+\ldots+f_{n-1}\left(c_{j, \ell}\right) v_{n}$

where $1 \leq j \leq m, 1 \leq \ell \leq n^{\prime}, c_{j, \ell} \in F_{2}$

$s_{m+1}=0$ over $F_{2^{n}} \Longleftrightarrow f_{0}=0, f_{1}=0, f_{n-1}=0$ over $F_{2}$. 


\section{Outline}

\section{Target - ECDLP \\ Background \\ Index Calculus Method with Gröbner Basis}

Our Contribution

\section{Symmetric function}

Using the fact that Semaev's summation polynomials are symmetric, substitute the polynomials with elementary symmetric functions.

For example :

$s_{3}=\left(x_{1} x_{2}+x_{1} x_{r}+x_{2} x_{r}\right)^{2}+x_{1} x_{2} x_{r}+\beta$ $=\left(\sigma_{2}+\sigma_{1} x_{r}\right)^{2}+\sigma_{2} x_{r}+\beta$

where

$\sigma_{1}=x_{1}+x_{2}, \sigma_{2}=x_{1} x_{2}, \beta$ is the parameter of $E_{\alpha, \beta}$. 


\section{Rewritten system for symmetric function}

- Variables rewritten

$$
\begin{aligned}
& x_{1}=c_{1,0} v_{1}+c_{1,1} v_{2}+\ldots+c_{1, n^{\prime}-1} v_{n^{\prime}} \\
& x_{2}=c_{2,0} v_{1}+c_{2,1} v_{2}+\ldots+c_{2, n^{\prime}-1} v_{n^{\prime}} \\
& \ldots \\
& x_{m}=c_{m, 0} v_{1}+c_{m, 1} v_{2}+\ldots+c_{m, n^{\prime}-1} v_{n^{\prime}}
\end{aligned}
$$

- Symmetric function rewritten

$$
\begin{aligned}
& \sigma_{1}=d_{1,0} v_{1}+d_{1,1} v_{2}+\ldots+d_{1, n-1} v_{n} \\
& \sigma_{2}=d_{2,0} v_{1}+d_{2,1} v_{2}+\ldots+d_{2, n-1} v_{n} \\
& \ldots \\
& \sigma_{m}=d_{m, 0} v_{1}+d_{m, 1} v_{2}+\ldots+d_{m, n-1} v_{n}
\end{aligned}
$$

- Relation of variables and symmetric function

$d_{1,0}=f_{1,0}\left(c_{i, j}\right)$

$d_{1,1}=f_{1,1}\left(c_{i, j}\right)$

..

$d_{m, n-1}=f_{m, n-1}\left(c_{i, j}\right)$

\section{Symmetric function}

- Using symmetric function for $s_{m+1}$ is not a new idea. Gaudry, Diem, Joux and Vitse proposed this in composite extension degree. $[2,3,4,5]$

- However, in prime extension degree makes the number of variables and number of polynomials grows too large. This makes it impracticable. 


\section{Special factor base $V$}

Let $F_{2^{n}}=F_{2}[\omega] / h(\omega)$, where $h(\omega)$ is an irreducible polynomial of prime degree $n$ over $F_{2}$.

Using the special factor base $V=\left\{1, \omega, \ldots, \omega^{n^{\prime}-1}\right\}$.

\section{Rewritten system for symmetric function}

- Variables rewritten

$$
\begin{aligned}
& x_{1}=c_{1,0}+c_{1,1} \omega+\ldots+c_{1, n^{\prime}-1} \omega^{n^{\prime}-1} \\
& x_{2}=c_{2,0}+c_{2,1} \omega+\ldots+c_{2, n^{\prime}-1} \omega^{n^{\prime}-1} \\
& \ldots \\
& x_{m}=c_{m, 0}+c_{m, 1} \omega+\ldots+c_{m, n^{\prime}-1} \omega^{n^{\prime}-1}
\end{aligned}
$$

- Symmetric function rewritten

$$
\begin{aligned}
& \sigma_{1}=d_{1,0}+d_{1,1} \omega+\ldots+d_{1, n^{\prime}-1} \omega^{n^{\prime}-1} \\
& \sigma_{2}=d_{2,0}+d_{2,1} \omega+\ldots+d_{2, n^{\prime}-2} \omega^{2 n^{\prime}-2} \\
& \ldots \\
& \sigma_{2}=d_{m, 0}+d_{m, 1} \omega+\ldots+d_{m, n^{\prime}-m} \omega^{n-m}
\end{aligned}
$$

- Relation of variables and symmetric function

$d_{1,0}=f_{1,0}\left(c_{i, j}\right)$

$d_{1,1}=f_{1,1}\left(c_{i, j}\right)$

$\cdots$

$d_{m, n^{\prime}-m}=f_{m, n^{\prime}-m}\left(c_{i, j}\right)$ 


\section{Symmetric function with specific vector base $V$}

\begin{tabular}{c||c|c|c}
\hline & $s_{m+1}$ & $s_{m+1}^{\prime}$ & $s_{m+1}^{\prime}$ with specific $\mathrm{V}$ \\
\hline \hline \#var & $m n^{\prime}$ & $m n^{\prime}+m n$ & $m n^{\prime}+\left(n^{\prime}-1\right) \frac{m(m+1)}{2}+m$ \\
\hline \#poly & $n$ & $n+m n$ & $n+\left(n^{\prime}-1\right) \frac{m(m+1)}{2}+m$ \\
\hline deg $_{\text {reg }}$ & 7 or 6 & 4 or 3 & 4 or 3 \\
\hline
\end{tabular}

Table: Comparison for different multivariate polynomial system

The time and memory costs are respectively roughly \#var $2 * d e g_{\text {reg }}$

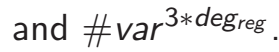

\section{Experimental Results}

CPU : AMD Opteron $6276^{*} 4,16$ cores, $2.3 \mathrm{GHz}$, L3 cache $16 \mathrm{MB}$ OS : CentOS 6.3

RAM : $512 \mathrm{~GB}$

Platform : Magma V2.18-9 64-bit version 


\section{Experimental Results}

Using Magma to finding one relation $\sum P_{i}=[a] P+[b] Q$.

\begin{tabular}{|c|c|c|c|c|c|c|c|c|c|}
\hline & \multirow{2}{*}{$\mathrm{n}$} & \multirow{2}{*}{$\mathrm{n}^{\prime}$} & \multicolumn{7}{|c|}{ sol: yes } \\
\hline & & & $\mathrm{D}_{\text {reg }}$ & var & poly & mono & $\mathrm{t}_{\text {trans }}$ & $t_{\text {groe }}$ & mem \\
\hline ImpFPPR & 23 & 3 & 6 & 9 & 23 & 2792.97 & 5.47 & 1.06 & 29.10 \\
\hline Imp Ours & 23 & 3 & 3 & 24 & 38 & 1079.60 & 0.91 & 1.04 & 15.59 \\
\hline Imp $\mathrm{p}_{F P R}$ & 53 & 3 & 6 & 9 & 53 & 6358.94 & 12.86 & 1.03 & 72.06 \\
\hline Impours & 53 & 3 & 3 & 24 & 68 & 2348.50 & 2.12 & 0.79 & 24.89 \\
\hline Imp ${ }_{F P P R}$ & 23 & $\overline{4}$ & 6 & 12 & 23 & 12059.19 & 21.06 & 6.83 & 95.66 \\
\hline Imp $p_{\text {Ours }}$ & 23 & 4 & 3 & 33 & 44 & 2173.29 & 1.83 & 3.19 & 29.63 \\
\hline ImpFPPR & 53 & 4 & 6 & 12 & 53 & 27655.34 & 50.63 & 1.86 & 272.55 \\
\hline Imp Ours & 53 & 4 & 3 & 33 & 74 & 4701.09 & 4.19 & 1.75 & 40.46 \\
\hline
\end{tabular}

Table: Comparison of the relation search $\left(m=3, n^{\prime}=3,4\right)$ with two strategies, ImpFPPR and Impours. Units are sec and MB

\section{Experimental Results}

Using Magma to finding one relation $\sum P_{i}=[a] P+[b] Q$.

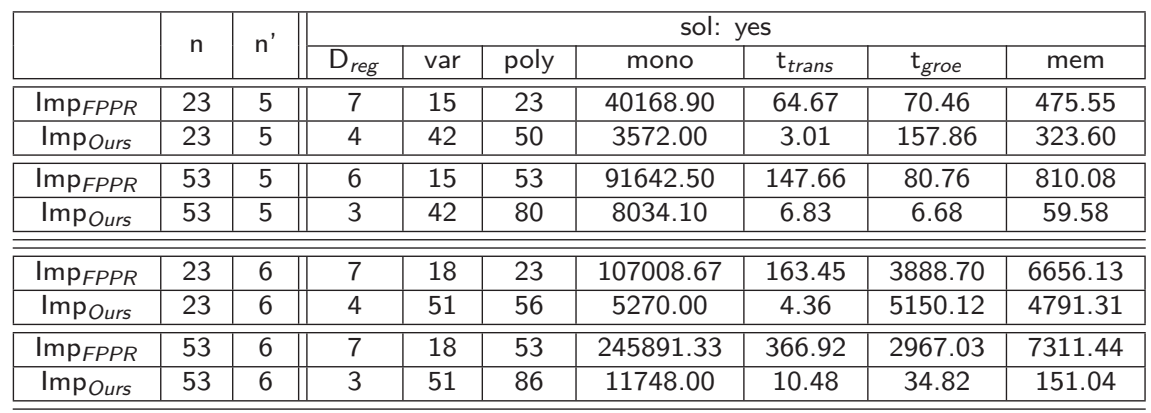

Table: Comparison of the relation search $\left(m=3, n^{\prime}=4,5\right)$ with two strategies, ImpFPPR and Impours. Units are sec and MB 


\section{Experimental Results}

Using Magma to solve ECDLP.

\begin{tabular}{c||c|c|c}
\hline$n$ & $\# E_{\alpha, \beta}$ & ImpFPPR & Imp Ours \\
\hline \hline 7 & $4 * 37$ & 1.574 & 0.864 \\
11 & $4 * 523$ & 8.625 & 6.702 \\
13 & $4 * 2089$ & 49.698 & 31.058 \\
17 & $4 * 32941$ & 2454.470 & 1364.742 \\
19 & $4 * 131431$ & 22474.450 & 9962.861 \\
\hline
\end{tabular}

Table: Comparison of two ECDLP strategies, ImpFPPR and Impours. The last two columns are computing time in seconds.

\section{Conlusion}

- This work has been accepted by IWSEC2013.

- We give the experimental evidence of our improvements.

- Future work - parallization. 


\section{Thanks}

\section{$\mathbf{Q} \& \mathbf{A}$}

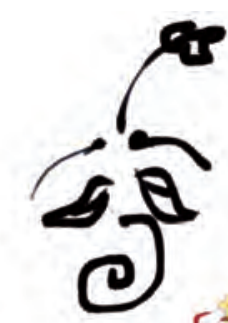

EP

\section{Reference I}

I. Semaev, "Summation polynomials and the discrete logarithm problem on elliptic curves," IACR Cryptology ePrint Archive, vol. 2004, p. 31, 2004.

C. Diem, "An index calculus algorithm for plane curves of small degree," in Hess et al. [14], pp. 543-557.

P. Gaudry, "Index calculus for abelian varieties of small dimension and the elliptic curve discrete logarithm problem," Journal of Symbolic Computation, vol. 44, no. 12, pp. 1690 - 1702, 2009

C. Diem, "On the discrete logarithm problem in elliptic curves," Compositio Mathematica, vol. 147, pp. $75-104,2011$

A. Joux and V. Vitse, "Elliptic curve discrete logarithm problem over small degree extension fields," Journal of Cryptology, pp. 1-25, 2011

C. Petit and J.-J. Quisquater, "On polynomial systems arising from a weil descent," in Advances in Cryptology ASIACRYPT 2012 (X. Wang and K. Sako, eds.), vol. 7658 of Lecture Notes in Computer Science, pp. 451-466, Springer Berlin Heidelberg, 2012

D. Hankerson, A. J. Menezes, and S. Vanstone, Guide to Elliptic Curve Cryptography. Secaucus, NJ, USA: Springer-Verlag New York, Inc., 2003.

I. Blake, G. Seroussi, N. Smart, and J. W. S. Cassels, Advances in Elliptic Curve Cryptography (London Mathematical Society Lecture Note Series).

New York, NY, USA: Cambridge University Press, 2005.

T. Saito, S. Yokoyama, T. Kobayashi, and G. Yamamoto, "Some relations between semaev's summation polynomials and stange's elliptic nets," Journal of Math-for-Industry, vol. 3 (2011A-9), pp. 89-92, 2011. 


\section{Reference II}

- R. P. Brent, "An improved monte carlo factorization algorithm," BIT Numerical Mathematics, vol. 20, pp. $176-184,1980$.

J. M. Pollard, "A monte carlo method for factorization," BIT Numerical Mathematics, vol. 15 (3), pp. 331-334, 1975

J.-C. Faugre, L. Perret, C. Petit, and G. Renault, "Improving the complexity of index calculus algorithms in elliptic curves over binary field," in Proceedings of Eurocrypt 2012, vol. 7237 of Lecture Notes in Computer Science, pp. 27-44, Springer Verlag, 2012.

J. H. Silverman, "The xedni calculus and the elliptic curve discrete logarithm problem," Designs, Codes and Cryptography, vol. 20, pp. 5-40, 1999.

F. Hess, S. Pauli, and M. E. Pohst, eds., Algorithmic Number Theory, 7th International Symposium, ANTS-VII, Berlin, Germany, July 23-28, 2006, Proceedings, vol. 4076 of Lecture Notes in Computer Science, Springer, 2006.

J.-C. Faugère, "A new efficient algorithm for computing gröbner bases $\left(\mathrm{f}_{4}\right)$," Journal of Pure and Applied Algebra, vol. 139, no. 1-3, pp. 61-88, 1999.

J. C. Faugère, "A new efficient algorithm for computing gröbner bases without reduction to zero $\left(f_{5}\right)$," in Proceedings of the 2002 international symposium on Symbolic and algebraic computation, ISSAC '02, (New York, NY, USA), pp. 75-83, ACM, 2002.

J. Faugère, P. Gianni, D. Lazard, and T. Mora, "Efficient computation of zero-dimensional gröbner bases by change of ordering," Journal of Symbolic Computation, vol. 16, no. 4, pp. 329 - 344, 1993.

\section{Reference III}

J. M. Pollard, "Kangaroos, monopoly and discrete logarithms," Journal of Cryptology, vol. 13, pp. 437-447, 2000.

D. Shanks, "Class number, a theory of factorization, and genera," in 1969 Number Theory Institute (Proc. Sympos. Pure Math., Vol. XX, State Univ. New York, Stony Brook, N.Y., 1969), pp. 415-440, 1971.

D. Bernstein, H.-C. Chen, C.-M. Cheng, T. Lange, R. Niederhagen, P. Schwabe, and B.-Y. Yang, "Ecc2k-130 on nvidia gpus," in Progress in Cryptology - INDOCRYPT 2010 (G. Gong and K. Gupta, eds.), vol. 6498 of Lecture Notes in Computer Science, pp. 328-346, Springer Berlin Heidelberg, 2010.

L. Judge, S. Mane, and P. Schaumont, "A hardware-accelerated ecdlp with high-performance modular multiplication," International Journal of Reconfigurable Computing, vol. 2012, 2012. 